\title{
Effects of Adding Respiratory Exercises to the Therapeutic Routine in Smartphone Users With Forward Head Posture and Non-Specific Chronic Neck Pain: A Randomised Controlled Trial
}

\author{
Hamid Rezaee Dareh-deh \\ Kharazmi University \\ Malihe Hadadnezhad \\ Kharazmi University \\ Amir Letafkar \\ Kharazmi University \\ Anneli Peolsson ( $\square$ anneli.peolsson@liu.se) \\ Linköping University https://orcid.org/0000-0002-6075-4432
}

\section{Research article}

Keywords: Therapeutic exercises, respiratory exercises, electromyography, forward head posture, chronic neck pain, smartphone users.

Posted Date: August 21st, 2020

DOI: https://doi.org/10.21203/rs.3.rs-53984/v1

License: (c) (i) This work is licensed under a Creative Commons Attribution 4.0 International License. Read Full License 


\section{Abstract}

Background: Neck pain and forward head posture are common in prolonged smartphone users and need to be targeted for treatment. We aimed to compare the effect of adding respiratory exercises to the therapeutic routine in smartphone users with forward-head posture and non-specific chronic neck pain.

Mehods: Sixty patients (aged 24.7 \pm 2.1 years), were randomly assigned to the therapeutic routine $(n=20)$, combined respiratory-exercises with therapeutic routine $(n=20)$, or control groups $(n=20)$. Each programme was implemented three times a week for 8 weeks. Forward head angle, pain, electromyographic activity, and respiratory patterns were measured at baseline and at 8-weeks' post-treatment.

Results: There were significant improvements in the combined group compared with the therapeutic routine group $(p=0.03)$ for diaphragm muscle activation, $(p=0.03)$, neck erector spinae activity $(p=0.04)$, respiratory balance $(p=0.04)$, and number of breaths $(p=0.02)$. There were significant within-group changes from baseline to post-treatment in the combined group for all outcomes above, but no changes in the therapeutic exercise routine group. Outcomes for the combined group were superior to the control group in every variable.

Conclusion: Combination treatment is more effective than the standard therapeutic routine. These results could be strengthened by more studies with longer follow-up assessments.

Trial registration: Current Controlled Trials using the IRCT website with ID number of, IRCT20200212046469N1

"Prospectively registered" at 2020/03/04.

\section{Background}

The use of electronic tools is increasing worldwide [1]. Varieties and the attractiveness of these tools have led to various groups of people, especially teenagers, using them for prolong periods and this has increased some related musculoskeletal problems [1]. It is commonly thought that in addition to psychological problems, like anxiety, headaches, insomnia, depression, poor sleep quality, and fatigue, long-term use of smartphones leads to inactivity and abnormal posture, such as the forward head posture (FHP) and rounded shoulders [2]. This can necessarily affect muscular activity, and place more pressure on the cervical spine where chronic pain originates $[3,4]$.

Individuals with FHP and chronic neck pain often suffer from weakness of the deep neck flexor muscles, which is to be compensated by excessive activity of other muscles such as sternocleidomastoid, and scalene [5]. This compensation leads to muscle imbalance and changes in the stress-strain diagram, by which cervical spine overload occurs $[5,6]$. This may also be observed in the thoracic spine as some involved muscles are connected to both areas. Also, disorders or impairments occur in the neck and respiratory system because of the joint activity of the above-mentioned muscles operated on the neck movements and respiratory function $[5,6]$.

Maximal inspiratory and expiratory pressure (MIP \& MEP) decrease in individuals with chronic neck pain and FHP [6]. This situation causes the respiratory pattern to change from nasal breathing to mouth breathing. As compared to the normal posture, both scalene and sternocleidomastoid muscles show a higher activity amount in FHP [5]. Such long-term activation can create poor respiratory habits to facilitate activities in auxiliary respiratory muscles [5]. Furthermore, the respiratory function is affected by changed muscle activity due to pain and disability, affecting the neck in a vicious circle. To improve such problems, various therapeutic and rehabilitative methods have been used such as the McKenzie exercise, Kinesio taping, and myofascial release [7]. Each method has demonstrated positive results in improving impairments and disorders in this area [7]. In this regard, some researchers have reported better consequences obtained from combinations of some of their therapeutic methods [7]. But previous research has not compared the effect of adding breathing exercises to therapeutic exercises; also respiratory exercises have shown benefits for respiration and 
balancing the main and auxiliary muscles [5]. Respiratory exercises are low cost and are also easily used in different situations [5]. In this regard, the present research aimed to compare the effect of adding respiratory exercises to the therapeutic routine on pain, electromyography (EMG), posture, and respiratory patterns in smartphone users with FHP and non-specific chronic neck pain. We hypothesised that adding respiratory exercise would enhance treatment effects on neck pain, respiratory pattern, electromyography, and posture in smartphone users with moderate chronic neck pain and FHP.

\section{Methods}

\subsection{Study Design and Participants}

The study was a randomised assessor-blind controlled trial (RCT ID: IRCT20200212046469N1). Patients (age $24.7 \pm 2.1$ years) with chronic neck pain were recruited by orthopaedic physicians via flyers displayed at the hospitals over 3-months from April to June 2020.

\section{Insert figure 1 here}

Inclusion criteria were males and females who using a smartphone for more than 4 hours a day who rated their 'worst pain over the last 24-hours' as moderate using the visual analogue scale (VAS), with neck disability index (NDI) scores between $28 \%$ and $45 \%$, and pain lasting longer than 3 months [8-11]. Forward head posture was defined as a cervical angle $<50^{\circ}$ [12-14]. A lateral-view photograph was taken to identify cervical angle in standing position [12-14].

The exclusion criteria were, previous history of neck or back surgery, neurological signs, rheumatoid arthritis, and currently using muscle relaxation medication. The local ethics committee at Kharazmi University approved the study protocol. This study complied with the ethical standards of the Declaration of Helsinki and all subjects signed informed consent.

Participants were randomised into two experimental groups and one control group, by drawing a number, from 1 to 63 , placed in sealed envelopes in a box prepared in advance by the trainer. The randomisation sequence was not disclosed until participants had completed their baseline assessments. The assessor was blinded to group allocation. Participants were not blinded to exercise study, however they were not aware which treatment was considered to be therapeutic. The same physiotherapist and trainer supervised both active treatment groups.

\subsection{Outcome Assessments}

The outcomes were measured at baseline and post-treatment two days after the 8-week intervention. The primary outcome measure was pain, and secondary outcome measures were EMG, respiratory pattern, and posture.

All participants completed a baseline questionnaire (Table 1). A physical therapy evaluation was performed by a Ph.D. trained physiotherapist with 25 years of clinical experience who was unaware of the type of treatments given and the group randomisation. All participants were instructed to limit their weekly exercise to the study treatment $[3,15,16]$.

\subsubsection{Pain intensity}

Pain was evaluated using VAS choosing a number from 0 (no pain at all) to 10 (unbearable pain), which was displayed along a horizontal line. This scale is widely used in clinical settings $[17,18]$ and is a valid and reliable tool [16]. The minimum clinically important difference for within-group on the pain scale has been reported to be 2.5-points in people with chronic neck pain with a baseline score greater than 6.0 [19].

\subsubsection{Electromyography}


An EMG device with eight channels (made by data Log Biometrics company, Canada) was used to measure the activity of upper trapezius muscles, sternocleidomastoid, scalene, neck erector spine, and diaphragm muscles. Based on the recommendations of SENIAM, the skin surface was shaved of hair and cleaned with alcohol swabs before wireless EMG electrodes were applied. EMG electrodes were placed in five areas as follows: upper trapezius, as positioned from the lateral to the midpoint, as an imaginary line was formed by the posterior aspect of the acromion and the spinous process of C7, and the electrode was placed on the muscle bulk [20]. For the sternocleidomastoid, the electrode was placed at the lower one-third of the line connecting the sternal notch and mastoid process [20]. Forward head posture was defined as a cervical angle $<50^{\circ}$ [12-14]. A lateral-view photograph was taken to identify cervical angles in a standing position [12-14]. For scalene muscles, the electrode was placed on the posterior triangle of the scalene muscle, above the clavicle, more inclined to the sternocleidomastoid (just posterior to and at a slightly oblique angle relative to the sternocleidomastoid [SCM], just above the clavicle and in the hollow triangle anterior of the upper trapezius) [21]. For neck erector spine, the electrode was attached to the muscles around the C4 vertebra [22]. The diaphragm, lower edge of the rib cage on a vertical line that passes through the nipple centre was selected for electrode placement [23].

The EMG information was collected using an EMG device with a sampling frequency of 1000 hertz, and in this study the EMG signal data were sampled at 1000 hertz. These signals were filtered in the bandpass between 20 and 500 hertz [20]. The full shoulder flexion task was used to obtain data on the activity of the selected muscles. In this regard, each flexion movement and return to the initial state was performed by the subjects at a 5-second time, in 3 consecutive times [20, 21, 24].

Additionally, to estimate a maximum voluntary contraction (MVC) for the upper trapezius, subjects placed their hand at $90^{\circ}$ abduction, sitting down on a chair, and were asked to apply pressure against the exposed resistance at the top [25]. To obtain the MVC for sternocleidomastoid and scalene muscles, subjects were placed in the supine position, and their hands were put on their own heads. Then, the head was anterolaterally placed and pressurised against the hand resistance [21]. To obtain the MVC for the erector spinae muscle, the subjects were asked to be in a prone posture and put both hands behind their head as moving the overhand against the resistance in the extension direction [25]. To achieve MVC for the diaphragm muscle in the sitting position subjects took deep breaths [26]. Each position of the maximal voluntary contraction was used two times for 5-second duration to normalise the data [25].

The EMG signal was processed by the Root Mean Square (RMS) algorithm in the MATLAB program. The resulting number represented the average power of a signal that indicated the muscle activity. To compare the subjects and normalise the data, the obtained values from the RMS were divided by those obtained from the MVC of each muscle, and the amount of muscle activity was considered as a percentage of the MVC $[20,21,24]$.

\subsubsection{Respiratory Pattern Assessment}

To assess the breathing pattern using Manual Assessment of Respiratory Motion (MARM), as the subject sitting on the bed, with natural breathing, the tester recorded and interpreted different aspects of respiration, including the number of breaths and the balance of respiration between the upper and lower parts of the rib cage and abdomen, by touching the lower back and lateral rib cage with both hands and without putting pressure on these parts [15].

\subsubsection{Forward head posture assessment}

The forward head and shoulder angles were measured using photogrammetry of the sagittal plane. This method favoured reliability, and it has been used in various research [3]. To measure the angles, three anatomical signs, including left tragus, acromion, and the spinous process of $\mathrm{C} 7$ vertebra were determined and marked. Then, the subjects were asked to stand at the designated area beside a wall (at a 23-cm distance) so that their left side was placed toward the wall. The photographic tripod supporting the digital camera was placed at a distance of $265 \mathrm{~cm}$, and its height was set 
based on the subject's right shoulder level. In such circumstances, the subjects were asked to lean forward three times and raise their hand over their head three times. They were then asked to stand in a completely relaxed and natural posture, and to look at an imaginary point on the opposite wall (eyes in line with horizon). The tester took images of the body profile view after a five-second pause. Finally, these images were transferred to a computer, and the angle of the line connecting tragus to $\mathrm{C} 7$ vertebrae, and that of the line connecting C7 and the acromion process were respectively measured with the vertical line (forward head and shoulder angles) using Kenova software (Kinova-0.8.27-64-bit, Kinova company, Canada) $[27,28]$.

\subsection{Training Protocol}

As intended in this study, training included two parts: therapeutic routine and respiratory exercises. The therapeutic exercises contained resistance and stretching exercises (in the three stretching exercises, we used the static stretching with a 30 -second hold for 2 sets) for 45 to 60 minutes per session, specifically one session a day for 3 sessions a week; totally all held in 8 weeks $[29,30]$. The rest interval between movements in these exercises was 45 and 30 seconds for resistance and stretching exercises respectively. In the combined group respiratory exercises were added to the therapeutic routine above which consisted of balloon breathing exercises performed in sessions of 4 sets, and as each set had 4 complete breathing breaks, these exercises were conducted for 2 sessions a day and 3 days a week for 8 weeks [31]. All exercise was done under supervision of a physical therapist at the pain clinic. All participants received documentation including information on postural corrections and improving general health.

\subsection{Control Group}

The control group $(n=20)$ received a pamphlet including information on postural corrections and improving general health during the 8-week study period. No other physical therapy modalities or treatments were performed [32].

\subsection{Statistical analysis}

The necessary sample size was estimated using G*Power 3.1.7 for Windows (G*Power@, University of Dusseldorf, Germany). The sample size calculation was considered a power calculation to detect between-group differences in the primary outcome measure (neck pain). To obtain $80 \%$ statistical power (1- $\beta$ error probability) with an a error level probability of 0.05 , we used repeated-measure analysis of variance (ANOVA), within-between interaction, and a medium effect size of 0.25 to consider two groups and two measurements for the primary outcome, generating a sample size about of 18 participants per group (total sample size of 54 subjects). The sample was increased to 63 (21 in each group) to allow for a $15 \%$ dropout rate. A total of 63 subjects met study criteria and participated in the study.

One-way ANOVA was used to compare the group demographics and post hoc independent t-tests were performed in the case of a significant omnibus test. The dependent variables of interest were pain, function, strength, and kinematics. For each variable, the 3-trial mean was calculated for each participant. One-way analysis of covariance (ANCOVA), with a between-factor of the group (control, therapeutic routine, combined exercises) and participants' baseline scores included as a covariate, was used to determine if there were group differences in the dependent variables of interest at posttesting.

This analysis approach (i.e. post-test performance as the outcome with baseline performance as a covariate) allowed us to compare post-testing outcomes while accounting for potential baseline group differences [33]. In the case of a significant omnibus test, pairwise comparisons were performed to examine potential between-group differences. These pairwise comparisons were based on the adjusted group means. In addition, 95\% confidence intervals (CI95\%) were calculated based on the adjusted group mean differences, and Cohen's d effect size (ES) statistics were calculated by dividing the adjusted group mean differences by the larger of the group standard deviations. The Bryant-Paulson procedure was used when conducting the pairwise comparisons and calculating the confidence intervals [34]. An alpha 
of .05 was used for all significance tests. Effect sizes of $0.2,0.5$, and 0.8 were considered 'small', 'moderate', and 'large' respectively [35]. SPSS software was used for statistical analysis (IBM Corp., Armonk, NY, USA).

\section{Results}

100 participants were recruited, 37 did not qualify based on the exclusion and inclusion criteria of the study, and 63 were randomised to 3 groups. Intention to treat protocol was followed for post-treatment analysis. See the CONSORT diagram for details (figure 1).

There was a high degree of adherence to all three interventions. Of the possible 24 sessions, the therapeutic routine group, the combined group, and the control group attended $20 \pm 2$ sessions, $20 \pm 1$ sessions, and $19 \pm 2$ sessions, respectively.

Our cohort comprised male and female subjects, mean age $24.7 \pm 2.1$ years, with $4.6 \pm 1.1$ pain on VAS at baseline and $46.4 \pm 3.4$ on the FHP. Further details on demographic data and additional baseline outcome measures are reported in table 1. Demographic characteristics and baseline outcome measures (age $(p=0.35)$, pain $(p=0.97)$, and FHP $(p=0.24))$ did not differ between the groups $(p>.05)$.

The main effects of group $\times$ time interactions are presented in Tables 2, 3, and 4.

For pain, at 8 weeks both the therapeutic routine $(E S=0.75, P=0.02)$ and combined groups $(E S=0.85, p=0.01)$ had significant within-group changes, but differences in the control group $(E S=0.21, p=0.38)$ were not significant. Differences between experimental groups were not significant $(\mathrm{ES}(95 \% \mathrm{Cl})=0.21(-0.13$ to 0.9$), \mathrm{p}=0.53)$ (table 2$)$.

For FHP, at 8 weeks both the therapeutic routine $(E S=-0.55, p=0.03)$ and combined groups $(E S=-0.70, p=0.01)$ had significant within-group changes, but differences in control group $(E S=0.12, p=0.43)$ were not significant. Differences between experimental groups were not significant ( $E S=0.17(0.41$ to 2.48$), p=0.206)$ (table 2$)$.

For amount of activity in the upper trapezius muscle, at 8 weeks the therapeutic routine (upper trapezius (ES $=0.81$, $p=0.02)$, sternocleidomastoid ( $E S=0.79, p=0.03)$, and scalene $(E S=0.86, p=0.02)$ ), and in combined (upper trapezius $(E S=0.95, p=0.01)$, sternocleidomastoid $(E S=0.91, p=0.01)$, scalene $(E S=0.92, p=0.01)$, neck erector spinae $(E S=0.90$, $p=0.01)$, and diaphragm $(E S=-0.78, p=0.01)$ groups had significant within-group changes, but differences in control group were not significant. Differences between experimental groups were significant in diaphragm muscle activation $(E S(95 \% \mathrm{Cl})=0.67(0.39$ to 1.31$), \mathrm{p}=0.03)$, and neck erector spinae $(\mathrm{ES}(95 \% \mathrm{Cl})=0.54(0.21$ to 0.92$), \mathrm{p}=0.03)($ table 3$)$.

The combined group had significant within-group changes in respiratory balance $(E S=-0.59, p=0.01)$, and the number of breaths $(E S=0.72, p=0.02)$, but differences in the control group, and therapeutic routine were not significant. Differences between experimental groups were significant in respiratory balance $(E S(95 \% \mathrm{Cl})=0.45(0.21$ to 0.87$), p=0.04)$, and in number of breaths $(E S(95 \% \mathrm{Cl})=0.54(0.17$ to 1.1$), \mathrm{p}=0.02)$ (table 4$)$.

\section{Discussion}

The present study aimed to compare the effect of adding respiratory exercises to therapeutic routine on pain, electromyography, posture, and breathing pattern in smartphone users with FHP and chronic non-specific neck pain. As with therapeutic routine exercises with or without breathing exercises, the results showed that the amount of pain and the FHP improved significantly after an 8-week relevant training intervention. However, no significant difference was observed between the two groups. 
Moreover, the amount of activity in the upper trapezius, sternocleidomastoid, scalene, and cervical erector spineae muscles revealed a significant decrease in both experimental groups, although no significant differences were found between these two groups. Having conducted the training interventions, the amount of activity in the diaphragm muscle indicated a remarkable increase in the combined group as compared with the two other groups, including the therapeutic and control ones. As for the respiratory pattern, a significant improvement and superiority were observed in the number of breaths and respiratory balance for the group of combined exercises compared to the therapeutic routine and control groups. In the control group, there were no significant differences over time.

Having performed the intended exercises, a decrease in activity could be found in the upper trapezius, sternocleidomastoid, scalene, and cervical erector spineae muscles in both exercise groups. However, changes in the diaphragm muscle activity were only observed in the combined group.

Regarding muscle activity the present results are consistent with Lee et al. [36] and Borisut et al. [37]. As the electronic tool being used in the FHP, the recent studies have indicated that increased activity was observed in the muscles, including the upper trapezius, sternocleidomastoid, cervical erector spineae, thoracic erector spineae, and neck extensors. Consequently, these muscles are shortened, and the deep cervical flexor muscles are weakened in such conditions [36]. In this regard, Borisut et al. [37] reported a decrease in the muscle activation of the cervical erector spineae, sternocleidomastoid, anterior scalene, and upper trapezius after strength exercises [37].

Having performed all the exercises in both experimental groups in the present study, the probable effect mechanism can be implied as activation in the collaborative muscles in the cervical area, correcting FHP, and decreasing the activity amount in muscles, including the upper trapezius, sternocleidomastoid, scalene, and cervical erector spineae [37]. In fact, adding the respiratory exercises to the therapeutic ones did not create a remarkable change in the activity of the upper trapezius, sternocleidomastoid, scalene, and cervical erector spineae muscles. However, a significant increase in diaphragm muscle activity was revealed due to the respiratory exercises in the combined group compared with the two other groups.

As for the FHP, the experimental groups indicated a considerable improvement as compared with the controls, but no significant difference was observed between the experimental groups. In this regard, the results obtained in this research were consistent with those found by Kong et al. [38]. According to the previous research, the chin-tuck exercise merely is not of enough durability [39]. Hence, researchers have tried to combine this exercise with some other endurance and strength exercises, aimed to strengthen the movement domain and increase the endurance of the cervical muscles. Kong et al. [38] reported that performing a course of modified cervical exercises has revealed a remarkable positive effect on the FHP of smartphone users who suffered from such an abnormal disorder. Therefore, the probable mechanism to improve the FHP in both experimental groups has been assumed to reduce the activity of upper trapezius, sternocleidomastoid, scalene, and cervical erector spinae muscles, strengthen cervical deep flexor muscles, and use collaborative muscles in this very area [38].

Before and after performance of the training interventions in the three involved groups, the number of pain changes were measured and evaluated using VAS. Although the controls experienced no significant reduction in pain, both experimental groups' subjects showed an observable decrease in pain following the interventions. Having reviewed the obtained results, no significant difference was found based on the effectiveness of pain between the two experimental groups.

As for the pain variable, the research results were consistent with those obtained from Chung et al.'s study [29] in which two exercise methods of 'craniocervical flexion and isometric neck exercise' were compared in patients with chronic neck pain in terms of the effect on pain. Their comparative results indicated that both mentioned exercise methods have had a remarkable effect in improving pain [29]. The present research aimed to compare the effect caused by adding respiratory exercises to the therapeutic routine by applying mere therapeutic exercises using a different method. Having conducted 
all the exercises, consequently, both experimental groups benefited from a remarkable improvement in their amount of pain while no significant difference was observed between both groups in this regard. As inferred from these findings, the addition of respiratory exercises did not increase the effect of the therapeutic ones on decreasing pain. Therefore, the positive effect of performed therapeutic exercises on balancing cervical muscle activity and improving posture in this area can be simply deemed the main cause to decrease pain.

On the other hand, the respiratory pattern was evaluated using the MARM method in this research, and the obtained results revealed that only the combined group experienced positive changes in this pattern as compared with the therapeutic routine. Generally, these results were consistent with those observed in Lee et al.'s study [40] Having examined whether and how a course of exercises affects the cervical angle and respiratory function in smartphone users, Lee et al. [40] reported that the participants conducted the related exercises, experienced positive and remarkable results in the cervical angle and multiple respiratory factors as compared with the controls [40]. As aimed in this research preface, the breath number and respiratory balance were compared to be affected by the respiratory-therapeutic and therapeutic exercises. The possible mechanism of effects caused by the respiratory-therapeutic exercises, which focused on correcting the FHP, seemed to strengthen respiratory muscles such as the diaphragm, increase lung volume, extend vital capacity and inform people with their body and breath position. Accordingly, the respiratory pattern was associated with positive changes in the respiratory-therapeutic exercise group [40, 41].

\section{Limitations}

Our study limitation was that no long-term follow-up assessment was considered in the current study, so a similar study with a follow-up stage is highly recommended. It is also recommended that future research use advanced respiratory assessment and respiratory exercise tools such as spirometry and power breath to assess other respiratory factors.

\section{Conclusions}

As compared with the therapeutic routine alone, performing 8-week respiratory-therapeutic exercises has positive effects on pain, FHP, and activity balance of the upper trapezius, sternocleidomastoid, scalene, and cervical erector spineae muscles, and also it can improve respiratory patterns. Moreover, diaphragm muscle activity is thereby enhanced to enable more complete breathing. Therefore, these exercises are recommended to correct upper quarter disorders.

\section{Abbreviations}

forward head posture (FHP), Maximal inspiratory and expiratory pressure (MIP \& MEP), electromyography (EMG), visual analogue scale (VAS), neck disability index (NDI), maximum voluntary contraction (MVC), Sternocleidomastoid (SCM), Root Mean Square (RMS), Manual Assessment of Respiratory Motion (MARM), analysis of variance (ANOVA), analysis of covariance (ANCOVA), 95\% confidence intervals (CI95\%), effect size (ES).

\section{Declarations}

\subsection{Ethics approval and consent to participate:}

This study was approved by the Research Ethics Committee of the Faculty of Physical Education and Sport Science of the Kharazmi University. The patients were informed about the details of the study and provided written informed consent before study enrolment. Informed consent was obtained from all the participants, and procedures were conducted according to the Declaration of Helsinki.

\subsection{Consent for publication}


Not applicable.

\subsection{Competing interests}

The authors declare that they have no competing interest.

\subsection{Funding}

None.

\subsection{Authors' contributions}

$\mathrm{MM}, \mathrm{AL}, \mathrm{HR}$, and AP developed the review protocol. $\mathrm{MH}$ and $\mathrm{HR}$ conducted search process and data extraction. All authors did quality appraisal of included studies and evidence synthesis as well as writing, reading and approving the final draft manuscript.

\subsection{Availability of data and materials}

The datasets analysed during the current study are available from the corresponding author on reasonable request.

\subsection{Acknowledgements}

The authors would like to thank all of the patients and health subject who took part in the study.

\section{References}

1. Jung SI, Lee NK, Kang KW, Kim K. The effect of smartphone usage time on posture and respiratory function. Phys Ther Sci. 2016; 28(3): 186-9.

2. Selvaganapathy K, Rajappan R, Dee TH. the Effect of Smartphone Addiction on Craniovertebral Angle and Depression Status Among University Students. Int J Integr Med. Sci. 2017; 4(7): 537-42.

3. Gadotti IC, Biasotto-Gonzalez DA. Sensitivity of clinical assessments of sagittal head posture. J Eval Clin Pract. 2010; 16(1): 141-4.

4. Alshahrani A, Aly SM, Abdrabo MS, Asiri FY. Impact of smartphone usage on cervical proprioception and balance in healthy adults. Biomed Res. 2018; 29(12): 2547-52.

5. Kang J-I, Jeong D-K, Choi $\mathrm{H}$. The effect of feedback respiratory exercise on muscle activity, craniovertebral angle, and neck disability index of the neck flexors of patients with forward head posture. Phys Ther Sci. 2016;28(9): $2477-81$.

6. Dimitriadis Z, Kapreli E, Strimpakos N, Oldham J. Respiratory weakness in patients with chronic neck pain. Man Ther. 2013;18(3): 248-53.

7. Kim J, Kim S, Shim J. Effects of McKenzie exercise, Kinesio taping , and myofascial release on the forward head posture. Phys Ther Sci. 2018; 30: 1103-7.

8. Fejer R, Kyvik KO, Hartvigsen J. The prevalence of neck pain in the world population: a systematic critical review of the literature. Eur Spine J. 2006;15: 834-848.

9. Bovim G, Schrader H, Sand T. Neck pain in the general population. Spine.1994; 19:1307-1309.

10. Blanpied Pr, Gross Ar, Elliott Jm, Devaney II, Clewley D, Walton DM, Sparks C, Robertson Ek. Neck Pain: Revision 2017 Clinical Practice Guidelines Linked to the International Classification of Functioning, Disability and Health from the Orthopaedic Section of the American Physical Therapy Association. J Orthop Sports Phys Ther. 2017;47(7):1-83.

11. Bartley EJ, Fillingim RB. Sex differences in pain: a brief review of clinical and experimental findings. Br J Anaesth. 2013; 111 (1): 52-8. 
12. Yip $\mathrm{CH}$, Chiu TT, Poon AT. The relationship between head posture and severity and disability of patients with neck pain. Man Ther. 2008; 13(2):148-54.

13. Lee KJ, Han HY, Cheon SH, et al.: The effect of forward head posture on muscle activity during neck protraction and retraction. Phys Ther Sci, 2015, 27: 977-979.

14. De-la-Llave-Rincón Al, Fernández-de-las-Peñas C, Palacios-Ceña D, et al. Increased forward head posture and restricted cervical range of motion in patients with carpal tunnel syndrome. J Orthop Sports Phys Ther, 2009, 39: 658-664.

15. Courtney R, Dixhoorn AEJ Van. Evaluation of Breathing Pattern: Comparison of a Manual Assessment of Respiratory Motion (MARM) and Respiratory Induction Plethysmography. App Psyc Bio. 2008; 33: 91-100.

16. Cleland JA, Childs JD, Whitman JM. Psychometric properties of the Neck Disability Index and numeric pain rating scale in patients with mechanical neck pain. Arch Phys Med Rehabil. 2008; 89: 69-74.

17. Kim D, Cho M, Park Y, et al. Effect of an exercise program for posture correction on musculoskeletal pain. Phys Ther Sci. 2015; 27: 1791-1794

18. Jensen MP, Karoly P, Braver S. The measurement of clinical pain intensity: a comparison of six methods. Pain. 1986; 27, 117-26.

19. Kovacs FM, Abraira V, Royuela A, Corcoll J, Alegre L, Tomás M, Mir MA, Cano A, Muriel A, Zamora J, Del Real MT, Gestoso M, Mufraggi N; Spanish Back Pain Research Network. Minimum detectable and minimal clinically important changes for pain in patients with nonspecific neck pain. BMC Musculoskelet Disord. 2008; 9:43.

20. K JW, S SM, L NK. Changes in upper-extremity muscle activities due to head position in subjects with a forward head posture and rounded shoulders. Phys Ther Sci. 2015; 27: 1739-42.

21. Bo-Been Kim, Ji-Hyun Lee, Hyo-Jung Jeong H-SC. Effects of suboccipital release with Craniocervical flexion exercise on Craniocervical alignment and extrinsic cervical muscle activity in subjects with forward head posture.

Electromyogr \& Kinesiol. 2016; 30: 31-37

22. Article O. Effect of duration of smartphone use on muscle fatigue and pain caused by forward head posture in adults. Phys Ther Sci. 2016; 28:1669-72.

23. Dds SV, Dds RM, Ignacia M, Bdent M, Dds HS, Dds CZ, et al. Awake teeth grinding in participants with canine guidance or group function: Effect on diaphragm EMG activity, heart rate, and oxygen saturation. J Cra SLE Pra. 2019; 00(00): 1-7.

24. Thigpen CA, Padua DA, Michener LA, Guskiewicz K, Giuliani C, Keener JD, et al. Head and shoulder posture affect scapular mechanics and muscle activity in overhead tasks. Electromyogr Kinesiol. 2010; 20(4):701-9.

25. Montakarn Chaikumarn NN\& PJ. Repeatability of EMG normalization of the neck and shoulder muscles in symptomatic office workers. Int J Occu Saf Ergo. 2017; 24: 422-30.

26. Walterspacher S, Pietsch F, Walker DJ, Röcker K, Kabitz HJ. Activation of respiratory muscles during respiratory muscle training. Res Phys Neur. 2018; 247: 126-32.

27. Shereen H. Elwardany, Wadida H. El-Sayed, Mohammad F. Reliability of Kinovea Computer Program in Measuring Cervical Range of Motion in Sagittal Plane. Ope Acc Lib J. 2015; 2: e1916.

28. Katherine Harman, PT, Cheryl L. Hubley-Kozey, Heather Butler. Effectiveness of an Exercise Program to Improve Forward Head Posture in Normal Adults: A Randomized, Controlled 10-Week Trial. Man Ther. 2005; 13(3): 163 - 176.

29. Chung S, Jeong YG. Effects of the craniocervical flexion and isometric neck exercise compared in patients with chronic neck pain: A randomized controlled trial. Phys The Pra. 2018; 34: 916-25.

30. Ruivo RM, Pezarat-correia P, Carita Al. Effects of a Resistance and Stretching Training Program on Forward Head and Protracted Shoulder Posture in Adolescents. Manipul Phys Ther. 2017; 40(1): 1-10. 
31. Boyle KL, Olinick J, Lewis C. The value of blowing up a balloon. North AmJ Sports Phys Ther. 2010; 5: 179-88.

32. Im B, Kim Y, Chung Y, Hwang S. Effects of scapular stabilization exercise on neck posture and muscle activation in individuals with neck pain and forward head posture. Phys Ther Sci. 2016; 28: 951-955.

33. Van Breukelen, GJP. ANCOVA versus change from baseline had more power in randomized studies and more bias in nonrandomized studies. J Clin Epid. 2006; 59: 920-925.

34. Vincent, WJ, Weir, JP. Statistics in Kinesiology. Champaign, IL: Human Kinetics, 2012; 266-268.

35. Cohen, J. A power primer. Psychol Bull 112: 155-159, 1992.

36. Lee S, Lee Y, Chung Y. Effect of changes in head postures during use of laptops on muscle activity of the neck and trunk. Phys Ther Rehab Sci. 2017; 33-8.

37. Borisut S, Vongsirinavarat M, Vachalathiti R, Sakulsriprasert P. Effects of Strength and Endurance Training of Superficial and Deep Neck Muscles on Muscle Activities and Pain Levels of Females with Chronic Neck Pain. Phys Ther Sci. 2013; 25(9): 1157-62.

38. Kong Y-S, Kim Y-M, Shim J. The effect of modified cervical exercise on smartphone users with forward head posture. Phys Ther Sci. 2017; 29(2): 328-31.

39. Thigpen CA, Lynch SS, Mihalik JP, Prentice WE, Padua D. The effects of an exercise intervention on forward head and rounded shoulder postures in elite swimmers. Br J Sports Med. 2010; 44(5): 376-81.

40. Lee NK, Jung SI, Lee DY, Kang KW. Effects of exercise on cervical angle and respiratory function in smartphone users. Oso Pub Heal Rese. Persp. 2017; 8: 271-4.

41. Se-Yoon Kim, Nan-Soo Kim. Laurentius Jongsoon Kim. Effects of cervical sustained natural apophyseal glide on forward head posture and respiratory function. Phys Ther Sci. 2015; 27: 1851-1854.

\section{Tables}

Table 1

Patient demographics at Baseline

\begin{tabular}{|lllll|}
\hline & $\begin{array}{l}\text { Control } \\
\text { Group }\end{array}$ & $\begin{array}{l}\text { Combined respiratory-therapeutic } \\
\text { exercise group }\end{array}$ & $\begin{array}{l}\text { Therapeutic exercises alone } \\
\text { Group }\end{array}$ & P \\
\hline Age, y & $25.3 \pm 1.4$ & $23.9 \pm 2.3$ & $24.9 \pm 2.8$ & 0.35 \\
\hline Height, $m$ & $177.9 \pm 4.5$ & $177.8 \pm 5.4$ & $177.0 \pm 5.7$ & 0.90 \\
\hline Weight, kg & $72.8 \pm 4.9$ & $71.8 \pm 6.0$ & $72.2 \pm 4.2$ & 0.90 \\
\hline $\begin{array}{l}\text { Body mass index, } \\
\text { kg/m }{ }^{2}\end{array}$ & $23.0 \pm 1.4$ & $22.6 \pm 1.1$ & $23.8 \pm 1.2$ & 0.62 \\
\hline FHP, Degree & $45.3 \pm 3.7$ & $46.5 \pm 2.3$ & $47.5 \pm 4.1$ & 0.24 \\
\hline Pain, VAS & $4.8 \pm 0.9$ & $5.1 \pm 1.6$ & $4.0 \pm 1.0$ & 0.97 \\
\hline Abbreviations: Visual analogue scale (VAS); forward head posture (FHP). & & \\
\hline
\end{tabular}


Table 2

Effect of training on pain and forward head posture.

\begin{tabular}{|c|c|c|c|c|c|c|c|c|}
\hline \multirow[b]{2}{*}{ Variable } & \multirow[b]{2}{*}{ Groups } & \multirow[b]{2}{*}{$\begin{array}{l}\text { Baseline } \\
\text { Mean } \pm \\
\text { SD }\end{array}$} & \multirow[b]{2}{*}{$\begin{array}{l}8- \\
\text { weeks } \\
\text { Mean } \\
\pm \text { SD }\end{array}$} & \multicolumn{3}{|c|}{ Within-Group } & \multicolumn{2}{|c|}{$\begin{array}{l}\text { Between- } \\
\text { groups }\end{array}$} \\
\hline & & & & $\begin{array}{l}\text { Change } \\
\text { Relative to } \\
\text { Baseline } \\
(\%)\end{array}$ & $\begin{array}{l}\text { Effect size }{ }^{\dagger} \text { and } \\
95 \% \text { Confidence } \\
\text { Interval (Lower limit } \\
\text {-Upper limit) }\end{array}$ & $p$ & $\begin{array}{l}\text { Interaction } \\
\text { Effects }\end{array}$ & $\mathrm{p}$ \\
\hline \multirow{4}{*}{$\begin{array}{l}\text { Pain, } \\
\text { VAS }\end{array}$} & \multirow{2}{*}{$\begin{array}{l}\text { Combined } \\
\text { respiratory- } \\
\text { therapeutic } \\
\text { exercise }\end{array}$} & \multirow[t]{2}{*}{$5.1 \pm 1.6$} & \multirow{2}{*}{$\begin{array}{l}1.9 \pm \\
0.6^{\ddagger}\end{array}$} & \multirow[t]{2}{*}{$62.4 \% \downarrow$} & \multirow[t]{2}{*}{0.85 (0.41 to 1.33$)$} & \multirow[t]{2}{*}{0.01} & \multirow{4}{*}{$\begin{array}{l}F=3.22 \\
P=0.04 \Omega\end{array}$} & \multirow{3}{*}{$\begin{array}{l}0.01 * \\
0.01 ¥\end{array}$} \\
\hline & & & & & & & & \\
\hline & $\begin{array}{l}\text { Therapeutic } \\
\text { exercise alone }\end{array}$ & $4.0 \pm 1.0$ & $\begin{array}{l}1.7 \pm \\
0.8^{\ddagger}\end{array}$ & $57.5 \% \downarrow$ & 0.75 (0.23 to 1.14$)$ & 0.02 & & \\
\hline & Control & $4.8 \pm 0.9$ & $\begin{array}{l}4.5 \pm \\
0.4\end{array}$ & $6.2 \% \downarrow$ & $0.21(-1.2$ to 0.03$)$ & 0.38 & & \\
\hline \multirow{4}{*}{$\begin{array}{l}\text { FHP, } \\
\text { Degree }\end{array}$} & \multirow{2}{*}{$\begin{array}{l}\text { Combined } \\
\text { respiratory- } \\
\text { therapeutic } \\
\text { exercise }\end{array}$} & \multirow{2}{*}{$\begin{array}{l}46.5 \pm \\
2.3\end{array}$} & \multirow{2}{*}{$\begin{array}{l}52.8 \pm \\
3.9^{\ddagger}\end{array}$} & \multirow[t]{2}{*}{$13.5 \% \uparrow$} & \multirow[t]{2}{*}{$-0.70(-2.5$ to -0.38$)$} & \multirow[t]{2}{*}{0.01} & \multirow{4}{*}{$\begin{array}{l}F=5.32 \\
P=0.03 \Omega\end{array}$} & \multirow{2}{*}{$\begin{array}{l}0.01 * \\
0.02 ¥\end{array}$} \\
\hline & & & & & & & & \\
\hline & $\begin{array}{l}\text { Therapeutic } \\
\text { exercise alone }\end{array}$ & $\begin{array}{l}47.5 \pm \\
4.1\end{array}$ & $\begin{array}{l}52.3 \pm \\
2.5^{\ddagger}\end{array}$ & $10.1 \% \uparrow$ & $-0.55(-1.92$ to -0.13$)$ & 0.03 & & \\
\hline & \multirow[t]{7}{*}{ Control } & $\begin{array}{l}45.3 \pm \\
3.7\end{array}$ & $\begin{array}{l}46.9 \pm \\
4.8\end{array}$ & $3.5 \% \uparrow$ & $-0.12(-0.08$ to 1.08$)$ & 0.43 & & \\
\hline & & \multicolumn{7}{|c|}{ *, Significant between combined respiratory-therapeutic exercise and control groups. } \\
\hline & & \multicolumn{7}{|c|}{$¥$, Significant between therapeutic exercise alone and control groups. } \\
\hline & & \multicolumn{7}{|c|}{$\S$, Percent change ( $\downarrow$ decrease, $\uparrow$ increase). } \\
\hline & & \multicolumn{7}{|c|}{$\begin{array}{l}\text { ‡, Denotes significant within group improvement between the baseline and 8-weeks } \\
\text { treatment period. }\end{array}$} \\
\hline & & \multicolumn{7}{|c|}{$\Omega$, Significant group $\times$ time interaction. } \\
\hline & & \multicolumn{7}{|c|}{ Abbreviations: Visual analogue scale (VAS); forward head posture (FHP). } \\
\hline
\end{tabular}


Table 3

Effect of training on muscle activation (\%MVC)

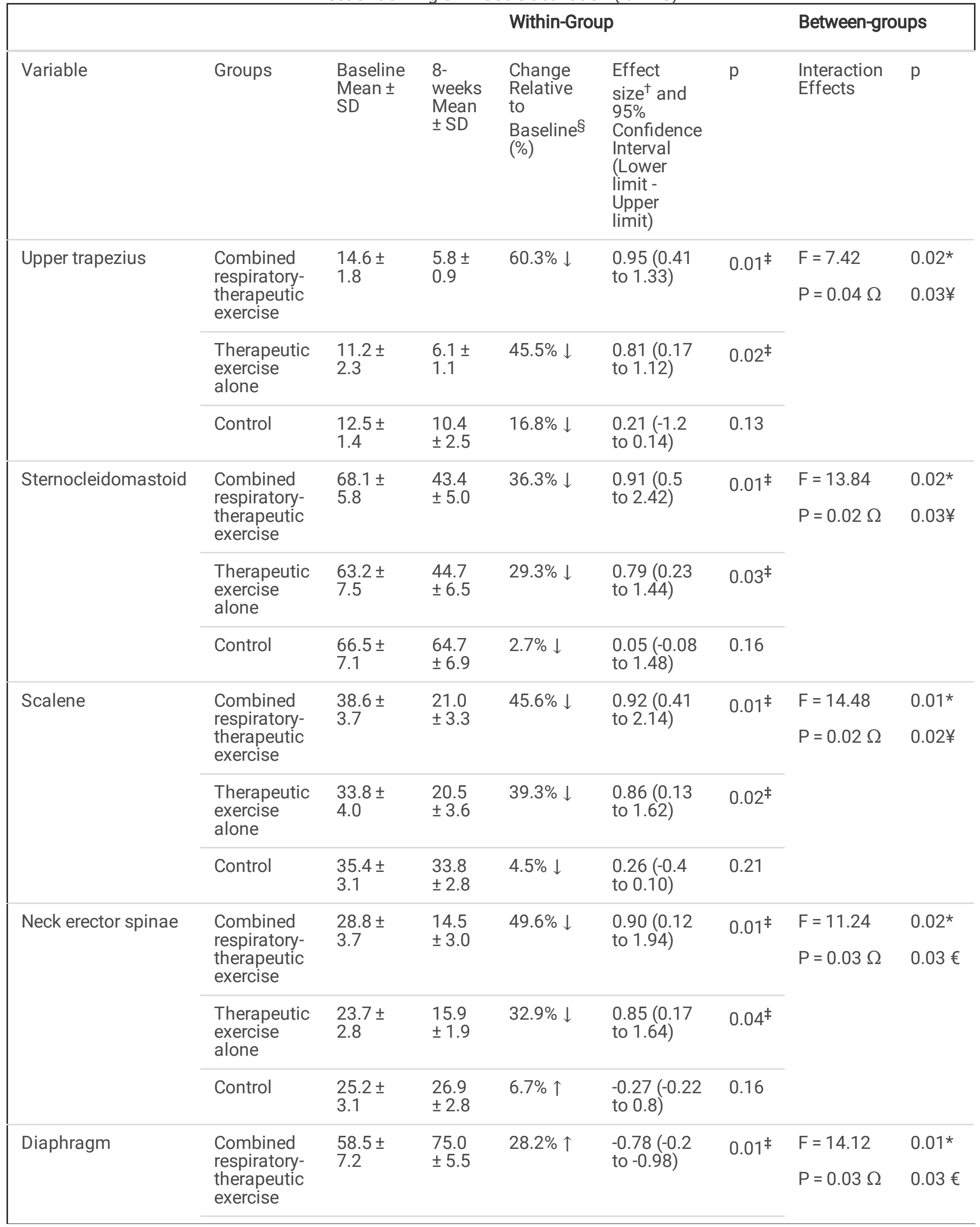




\begin{tabular}{|c|c|c|c|c|c|}
\hline & & \multicolumn{3}{|c|}{ Within-Group } & \multirow[t]{2}{*}{ Between-groups } \\
\hline $\begin{array}{l}\text { Therapeutic } \\
\text { exercise } \\
\text { alone }\end{array}$ & $\begin{array}{l}61.1 \pm \\
6.7\end{array}$ & $\begin{array}{l}58.4 \\
\pm 7.9\end{array}$ & $4.4 \% \downarrow$ & $\begin{array}{l}0.18(-0.2 \\
\text { to } 0.46)\end{array}$ & \\
\hline Control & $\begin{array}{l}55.4 \pm \\
5.3\end{array}$ & $\begin{array}{l}53.8 \\
\pm 6.4\end{array}$ & $2.9 \% \downarrow$ & $\begin{array}{l}0.13(-0.12 \\
\text { to } 0.07)\end{array}$ & 0.22 \\
\hline \multicolumn{6}{|c|}{ *, Significant between combined respiratory-therapeutic exercise and control groups. } \\
\hline \multicolumn{6}{|c|}{$¥$, Significant between therapeutic exercise alone and control groups. } \\
\hline \multicolumn{6}{|c|}{$\S$, Percent change ( $\downarrow$ decrease, $\uparrow$ increase). } \\
\hline \multicolumn{6}{|c|}{$\ddagger$, Denotes significant within group improvement between the baseline and 8-weeks treatment period. } \\
\hline \multicolumn{6}{|c|}{$€$, Significant between combined respiratory-therapeutic exercise and therapeutic exercise alone groups. } \\
\hline \multicolumn{6}{|l|}{$\Omega$, Significant group $\times$ time interaction. } \\
\hline
\end{tabular}


Table 4

Effect of training on respiratory pattern.

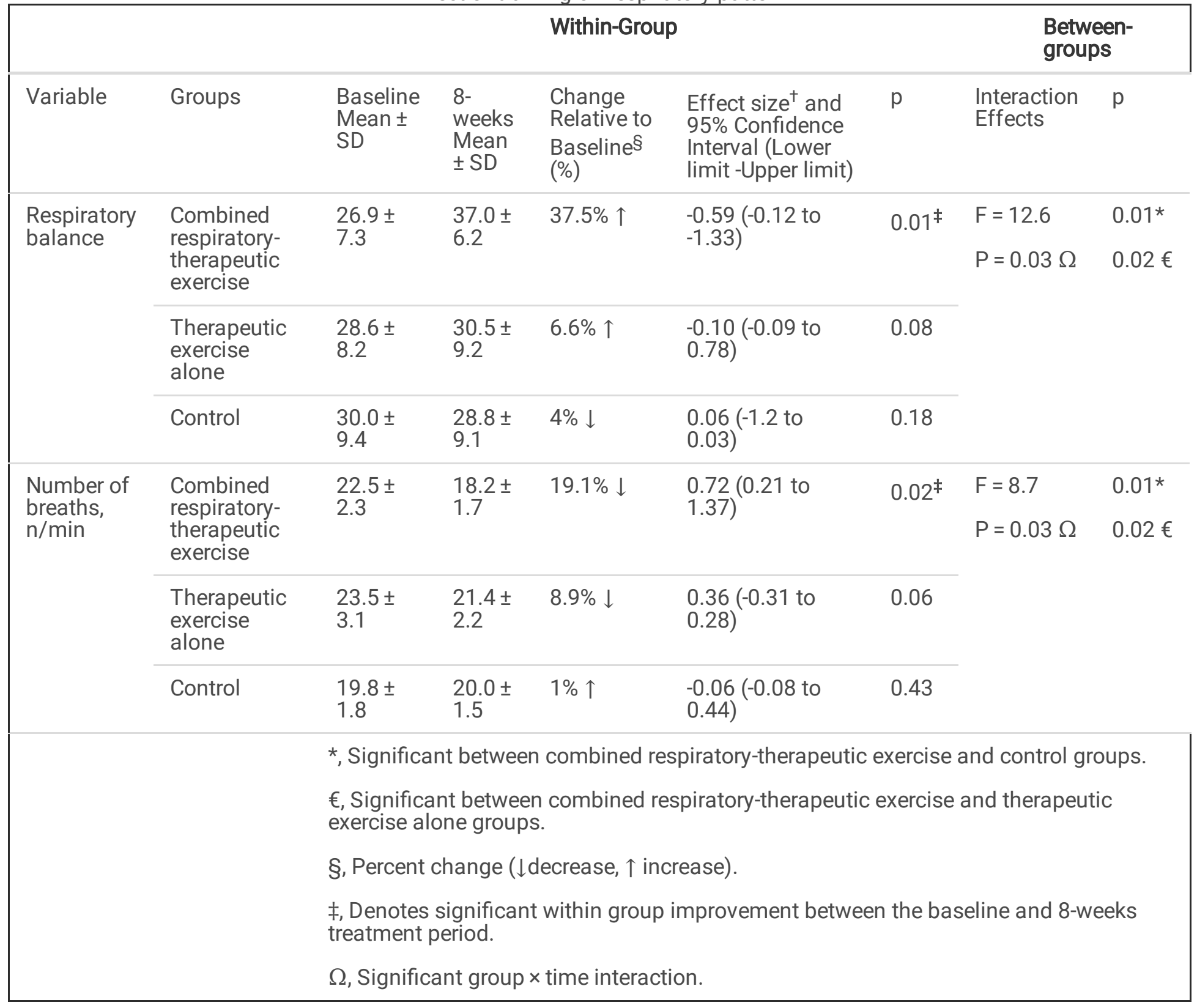

\section{Figures}




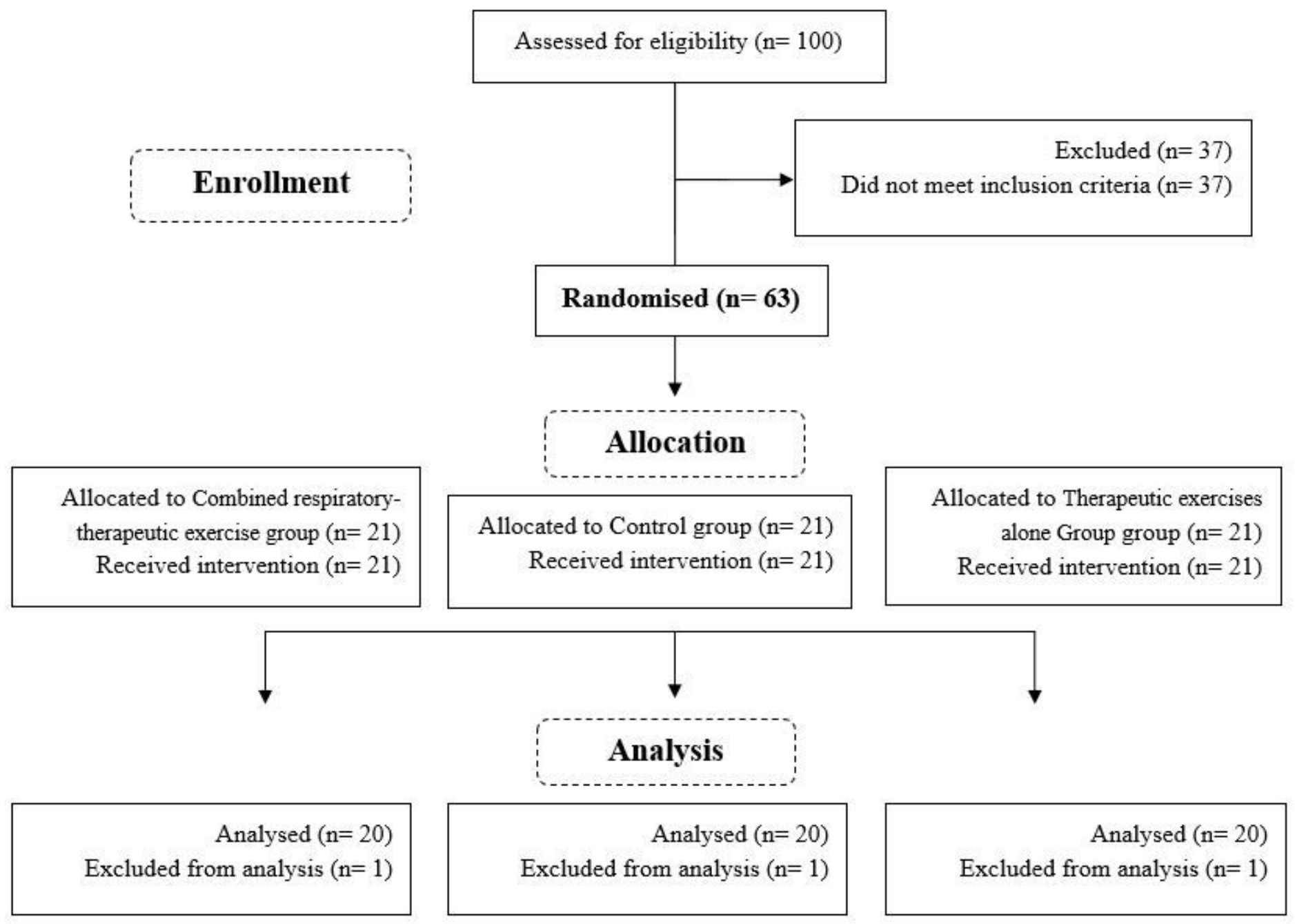

Figure 1

Flow Diagram of the study

\section{Supplementary Files}

This is a list of supplementary files associated with this preprint. Click to download.

- CONSORT2010ChecklistMSWord.doc 